\title{
Modeling and Analysis of DIPPM: A New Modulation Scheme for Visible Light Communications
}

\author{
Sana Ullah Jan, Young-Doo Lee, and Insoo Koo \\ Department of Electrical Engineering, University of Ulsan, Ulsan 680-749, Republic of Korea \\ Correspondence should be addressed to Insoo Koo; iskoo@ulsan.ac.kr
}

Received 19 March 2015; Revised 16 June 2015; Accepted 25 June 2015

Academic Editor: Geoffrey A. Cranch

Copyright (C) 2015 Sana Ullah Jan et al. This is an open access article distributed under the Creative Commons Attribution License, which permits unrestricted use, distribution, and reproduction in any medium, provided the original work is properly cited.

\begin{abstract}
Visible Light Communication (VLC) uses an Intensity-Modulation and Direct-Detection (IM/DD) scheme to transmit data. However, the light source used in VLC systems is continuously switched on and off quickly, resulting in flickering. In addition, recent illumination systems include dimming support to allow users to dim the light sources to the desired level. Therefore, the modulation scheme for data transmission in VLC system must include flicker mitigation and dimming control capabilities. In this paper, the authors propose a Double Inverse Pulse Position Modulation (DIPPM) scheme that minimizes flickering and supports a high level of dimming for the illumination sources in VLC systems. To form DIPPM, some changes are made in the symbol structure of the IPPM scheme, and a detailed explanation and mathematical model of DIPPM are given in this paper. Furthermore, both analytical and simulation results for the error performance of 2-DIPPM are compared with the performance of VPPM. Also, the communication performance of DIPPM is analyzed in terms of the normalized required power.
\end{abstract}

\section{Introduction}

The demand for additional bandwidth in mobile communications is growing day by day due to the significant increase in the number of mobile users, and the radio capacity of current systems is almost fully occupied. Since it has become quite difficult to find available radio capacity to fulfill demand for wireless data transmissions for media applications [1], Visible Light Communication (VLC) has been developed as an alternate to communication over radio frequencies.

VLC refers to wireless communications that use the visible light spectrum (380 to $780 \mathrm{~nm}$ ) as the transmission medium [2]. VLC systems transmit data by continuously turning light sources (LEDs) on and off to conduct intensity modulation. The receiver consists of a photodetector that receives data via direct detection. The light source is also used for illumination, its primary function, in addition to data transmission. The dual use of both illumination and data transmission involves two main challenges for VLC: flicker mitigation and dimming control.

Flickering refers to the fluctuation in the brightness of the light perceived by the naked human eye [3]. This occurs as a result of the variation in the brightness of light due to repeated on/off switching of the light source. The IEEE 802.15.7 standard differentiates flickering into intraframe flickering, which is the perceivable fluctuation in brightness within a frame, and interframe flickering, which is the perceivable fluctuation in brightness between adjacent frames [2]. Flickering must be mitigated because it is harmful for human eye $[2,3]$.

In addition, modern lighting systems provide dimming support to allow users to control the average brightness level of the light source. Dimming support is also important for VLC due to the potential for energy savings and increased energy efficiency [2].

Flicker mitigation and dimming control can be achieved through an appropriate modulation scheme. Various modulation schemes for VLC have been proposed that take both of these challenges into account. For example, the IEEE 802.15.7 standard proposes on-off Keying (OOK), Variable Pulse Position Modulation (VPPM), and Color Shift Keying (CSK) modulation schemes [2]. In [4], Lee and Park presented a comparison of the performance between OOK, VPPM, and Multiple Pulse Position Modulation (MPPM) schemes. In [3], 


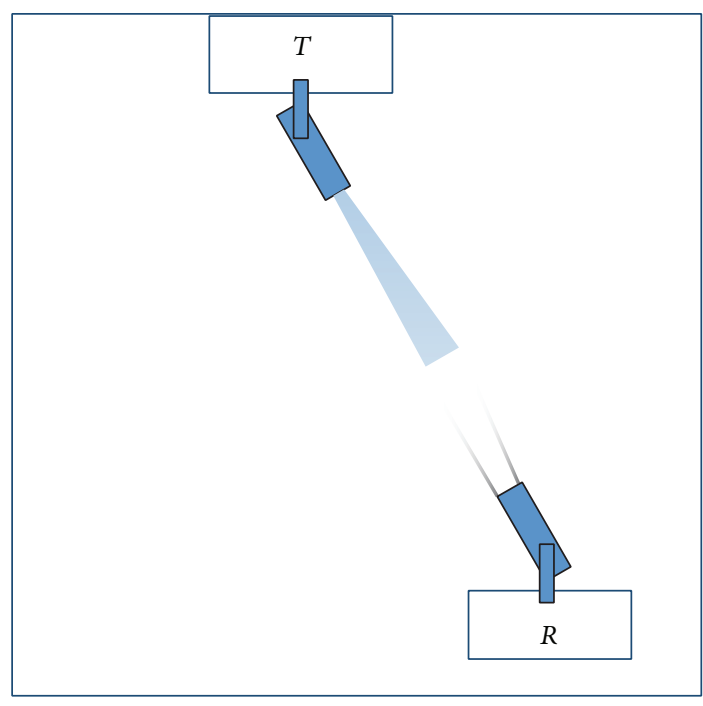

(a)

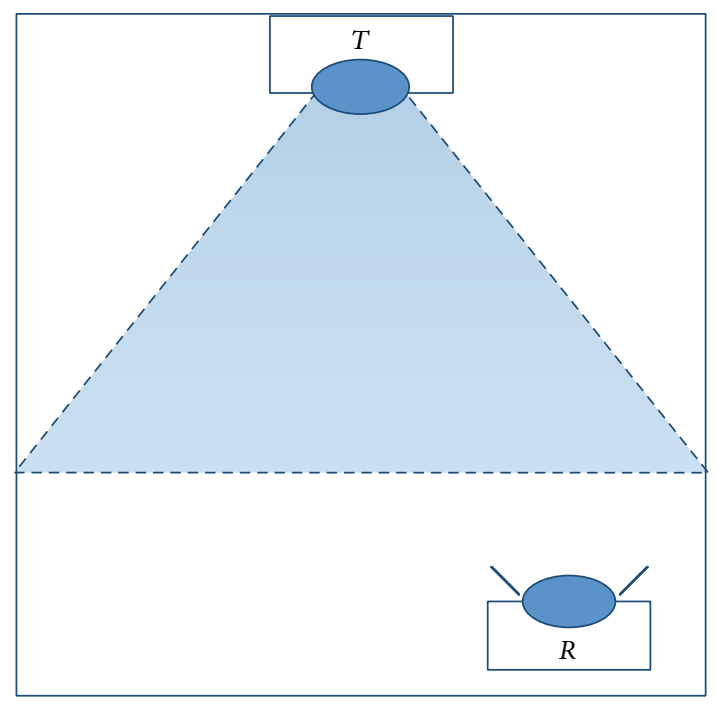

(b)

FiguRE 1: VLC link configuration: (a) directed LOS, (b) nondirected LOS.

Oh proposed a Pulse Dual Slope Modulation (PDSM) scheme to mitigate flicker by changing the slope of the rising and falling edges of the pulse to represent 1 and 0 bits, respectively. In [5], Choi et al. merged CSK and VPPM schemes to change the ratio of the colors (red, green, and blue), which changes the perceivable color of light, while the pulse width controls dimming.

In this paper, the authors propose a Double Inverse Pulse Position Modulation (DIPPM) scheme that allows for both flicker mitigation and dimming control. DIPPM is a modified form of the Inversed Pulse Position Modulation (IPPM) scheme. DIPPM symbol is obtained by inserting a compressed IPPM symbol in the off slot of IPPM symbol. The data is encoded by changing the position of the off slot, allowing for data transmission of more than one bit per symbol. We analyzed the performance of 2-DIPPM for a VLC system with a Line-of-Sight (LOS) channel. The analytical results and the simulation indicate that our proposed approach is effective at higher dimming levels.

This paper is organized as follows. In Section 2, the VLC system model for DIPPM is described. In Section 3, a generalized mathematical model and the symbol structure of DIPPM are shown and, also, the performance analysis is given in terms of bit error rate and average power of DIPPM. In Section 4, simulation results are given where BER performance of 2-DIPPM is compared with that of VPPM and average power of DIPPM is shown. Finally, the paper is concluded in Section 5.

\section{System Model}

The VLC link can be configured in many ways depending on the system configurations $[1,6]$. The two categories are directed LOS and nondirected LOS channels as shown in Figure 1. In directed LOS system, the light is concentrated in a very narrow beam as shown in Figure 1(a). This type of system is normally used for point-to-point communication. The nondirected LOS channel uses wide beam transmitters and wide field of view (FOV) receivers as shown in Figure 1(b). In the paper, we consider a nondirected LOS VLC system which is normally used for indoor applications. A LED light is installed in the ceiling of the room, as a transmitter. The LED light is used for data transmission in addition for the illumination. A photodetector is used as a receiver. The photodetector's azimuth angle and elevation angle are assumed such that the transmitter is inside the photodetector's FOV. The azimuth angle is the angle between the $x$-axis and the projection of the transmitter receiver line on the $x-y$ plane as shown in Figure 2(a). The transmitter's position is given by vector $\left(X^{\prime}, Y^{\prime}, Z^{\prime}\right)$ from the receiver's position $(0,0,0)$. The projection of the transmitter-receiver line on $x-y$ plane is given by the vector $\left(X^{\prime}, Y^{\prime}, 0\right)$. The angle " $A$ " between the $x$-axis and vector $\left(X^{\prime}, Y^{\prime}, 0\right)$ represents the azimuth angle. The elevation angle is defined by receiver orientation with $x$ axis as shown in Figure 2(b). Here "n" is the normal vector to the receiver surface. The angle " $h$ " between $x$-axis and the receiver surface' normal " $\mathbf{n}$ " is the elevation angle. The receiver pointing straight up has an elevation angle of $90^{\circ}$ while the source pointing straight down has an elevation angle of $-90^{\circ}[7,8]$. Therefore, in the paper we assume that there exists at least one direct path of transmission from transmitter to the receiver, such that the receiver can receive the light through the direct path from transmitter without reflecting from any obstacle and also from the indirect path with light reflected from obstacles and walls of the room. Subsequently, a nondirected LOS VLC channel is considered in this paper.

The overall block diagram of the VLC system being considered is given in Figure 3. We utilize the system model that Yoo and Jung applied for VPPM scheme in [9] such that we adopt the same mathematical equations of the system model for our proposed scheme. The transmitted DIPPM 


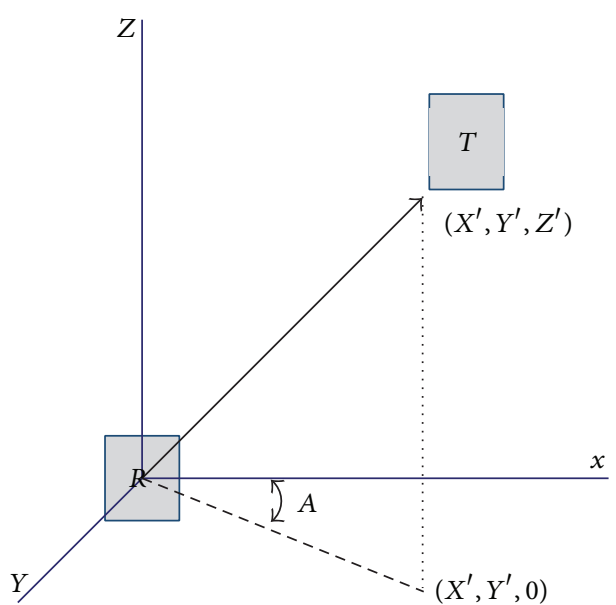

(a)

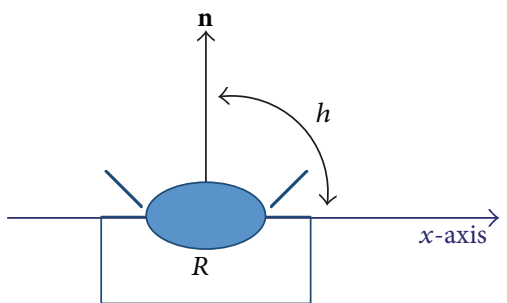

(b)

Figure 2: (a) Azimuth angle. (b) Elevation angle.

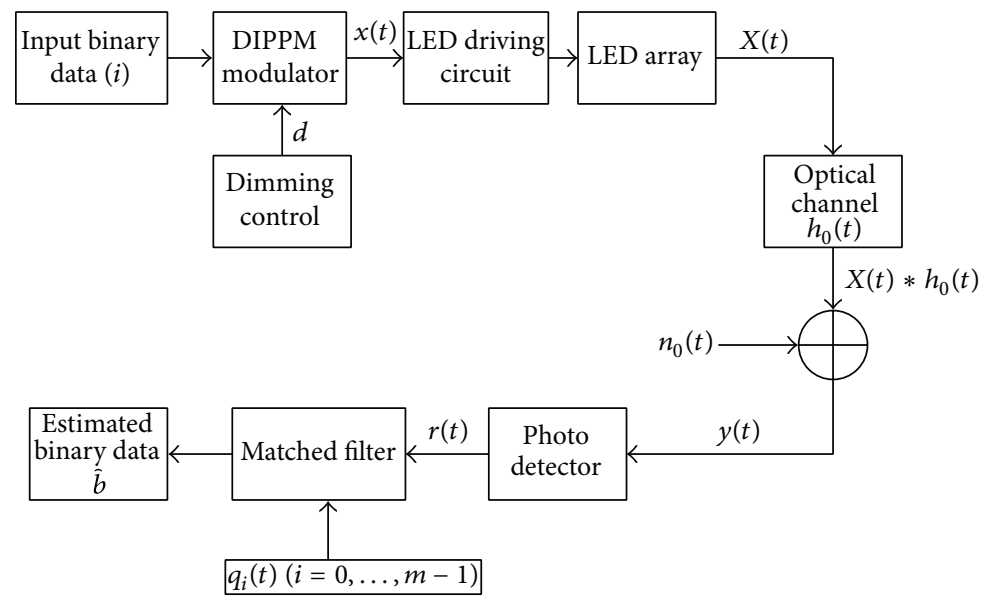

FIGURE 3: Block diagram of VLC system.

modulated signal, which is obtained by passing the binary input signal (data) through a DIPPM modulator with a dimming control, is expressed as

$$
x(t)=\sum_{j=-\infty}^{\infty} s\left(t-j T_{s}\right),
$$

where $s(t)$ denotes the modulated signal with symbol duration $T_{s}$.

The optical signal, denoted by $X(t)$, is transmitted through the light source. The received optical signal, denoted by $y(t)$, is given as

$$
y(t)=X(t) * h_{0}(t)+n_{0}(t),
$$

where $h_{0}(t)$ is the optical channel gain, " $*$ " is the convolution sign, and $n_{0}(t)$ is the optical noise.
The matched filter receives the signal $r(t)$ after the photodetector as follows:

$$
\begin{aligned}
r(t) & =R y(t)=R\left(X(t) * h_{0}(t)+n_{0}(t)\right) \\
& =H(0) x(t) * h(t)+n(t),
\end{aligned}
$$

where $R$ is the photodetector conversion responsivity, $H(0)$ indicates the path loss gain of the signal, $h(t)$ denotes the electrical impulse response of the optical wireless channel, and $n(t)$ is the electrical additive white Gaussian noise with zero mean and variance $N_{0} / 2$.

The electrical impulse response is given as

$$
h(t)=\sum_{k=0}^{C} G_{k} \exp \left(-\tau_{k}\left(t-t_{d, k}\right)\right) u(t),
$$

where $C$ represents the number of channel clusters and $G_{k}, \tau_{k}$, and $t_{d, k}$ are the channel gain, time constant, and time delay of the $k$ th cluster, respectively. Finally, the transmitted data is 


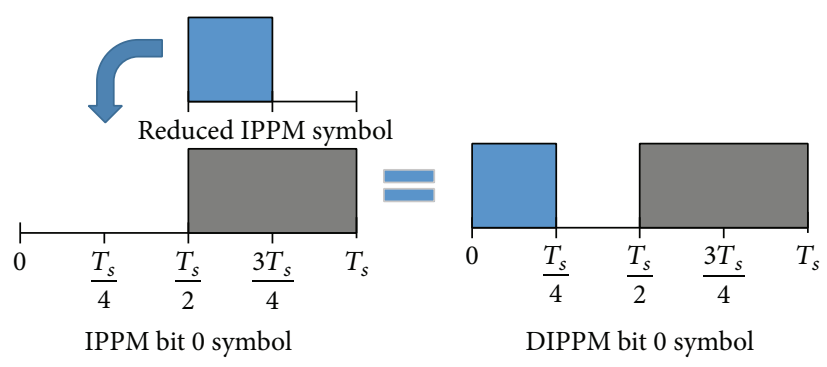

(a)

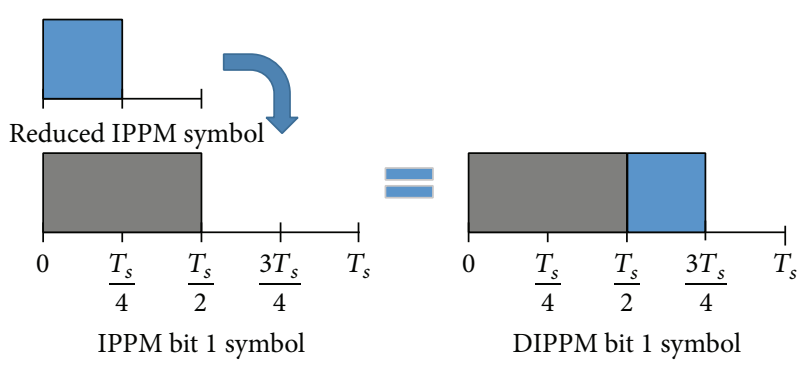

(b)

FIGURE 4: DIPPM signaling structure of 2-DIPPM $(n=1)$ for $(a)$ the bit 0 case and $(b)$ the bit 1 case.

estimated by using a maximum likelihood (ML) decision rule given by [10]

$$
\widehat{b}=\arg \max _{i=0,1, \ldots, m-1} r_{i}
$$

where $r_{i}$ is the $i$ th element of possible output signal vectors space $r=\left[r_{0}, r_{1}, \ldots, r_{m-1}\right]$.

\section{The Proposed Double IPPM (DIPPM)}

The $m$-DIPPM inserts a reduced IPPM (R-IPPM) symbol of duration $T_{s} / m$ into the off slot of the IPPM symbol with duration $T_{s}$. The R-IPPM symbol of duration $T_{s} / m$ has an off slot duration of $T_{s} / m^{2}$. The $m$-DIPPM transmits $n\left(=\log _{2} m\right)$ bits per symbol. The DIPPM symbol structures for one bit per symbol $(n=1)$ are shown in Figure 4. This figure shows how an IPPM symbol can be modified to get the proposed DIPPM modulation scheme. The $L$-IPPM symbol divides the symbol duration into $L$ equal time intervals. One of the $L$ intervals has an off slot while keeping all other slots on. Thus the information is sent by changing the position of the off slot. In the case of 1 bit per symbol $(L=2)$, the whole symbol duration is divided into two slots each of duration $T_{s} / 2$. In Figure 4(a), the IPPM bit 0 symbol is assumed which has an off slot interval from 0 to $T_{s} / 2$. To obtain DIPPM symbol, a reduced IPPM symbol which has a total duration of $T_{s} / 2$ and duty cycle $T_{s} / 4$ is inserted in the off slot of the IPPM symbol, which becomes the bit 0 symbol of DIPPM, and also results in increase in the duty cycle from $T_{s} / 2$ to $3 T_{s} / 4$. If the same reduced IPPM symbol is inserted in the off slot of bit 1 IPPM symbol, the bit 1 symbol of DIPPM can be obtained as shown in Figure 4(b). It is noteworthy that any of the two reduced IPPM symbols can be selected for forming DIPPM symbol. That is, the reduced IPPM symbol with first half off slot and second half on slot or the symbol with first half on slot and second half off slot can be used for forming DIPPM symbol. However, the chosen reduced IPPM symbol should be used throughout the system after being chosen.

The $m$-DIPPM modulated signal is given as

$$
s(t)=P \varphi_{i}(t),
$$

TABLE 1: Duty cycle for different bit resolutions per symbol.

\begin{tabular}{lc}
\hline$n$ & $\delta$ \\
\hline 1 & $75 \%$ \\
2 & $93.75 \%$ \\
3 & $98.44 \%$ \\
4 & $99.61 \%$ \\
\hline
\end{tabular}

where $P$ is the instantaneous power and $\varphi_{i}(t)$ is the basis function with $i$ as the decimal value of $n$ bits per symbol of input data. The instantaneous power is expressed as

$$
P=\sqrt{2 d E_{s}}
$$

where $d \in(0,1)$ is the required dimming level and $E_{s}$ is the symbol energy.

The mathematical expression of the basis function, $\varphi_{i}(t)$, is given as follows:

$$
\varphi_{i}(t)= \begin{cases}0, & \frac{a T_{s}}{m^{2}} \leq t \leq \frac{(a+1) T_{s}}{m^{2}}, \\ \sqrt{\frac{1}{\delta T_{s}},} & \text { otherwise, }\end{cases}
$$

where $T_{s}$ is the symbol duration, $m=2^{n}$ for $n$ bits per symbol, $\delta$ is the duty cycle, and the value of $a$ depends on the bit resolution. The value of $a$ for $m$-DIPPM is given as follows:

$$
\begin{aligned}
& a=1 \quad \text { for } i=0, \\
& a=1+m \text { for } i=1, \\
& a=1+2 m \text { for } i=2, \\
& \vdots \\
& a=1+(m-1) m \text { for } i=m-1 .
\end{aligned}
$$

The basis function is normalized to have a unit energy of $\int_{0}^{T_{s}} \varphi_{i}^{2}(t) d t=1$. The duty cycle of the symbol is given as

$$
\delta=\frac{m^{2}-1}{m^{2}} .
$$

The duty cycles for different bit resolutions are given in Table 1. 


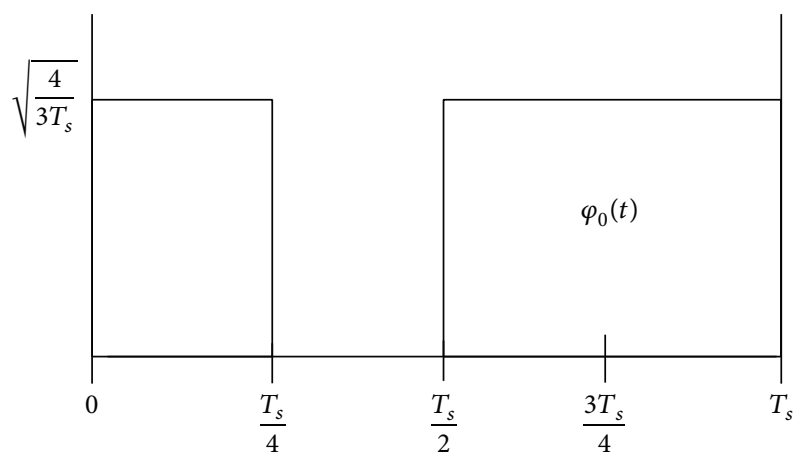

(a)

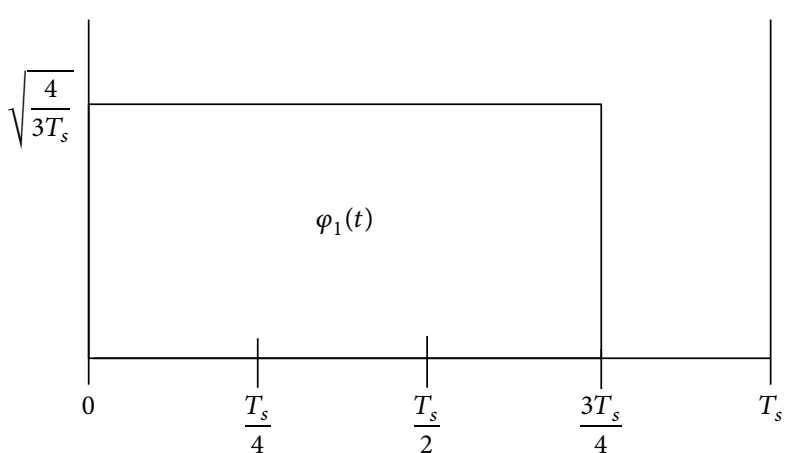

(b)

FIGURE 5: 2-DIPPM symbol structure for (a) data bit 0 and (b) data bit 1 .

3.1. Bit Error Rate (BER) Analysis for 2-DIPPM. In this subsection, we analyze the bit error rate (BER) performance in the case of 2-DIPPM. The 2-DIPPM $(n=1)$ modulated signal is given according to (6) such that

$$
s(t)= \begin{cases}P \varphi_{0}(t), & i=0, \\ P \varphi_{1}(t), & i=1,\end{cases}
$$

where $\varphi_{0}(t)$ and $\varphi_{1}(t)$ represent the basis functions for data bits 0 and 1 , respectively.

The values of $a$ for 2-DIPPM are obtained using (9) as follows:

$$
\begin{array}{ll}
a=1 & \text { for } i=0, \\
a=3 & \text { for } i=1 .
\end{array}
$$

The basis functions can be derived from (8) and (12) as follows:

$$
\begin{gathered}
\varphi_{0}(t)= \begin{cases}0, & \frac{T_{s}}{4} \leq t \leq \frac{T_{s}}{2}, \\
\sqrt{\frac{4}{3 T_{s}},} & \text { otherwise, }\end{cases} \\
\varphi_{1}(t)= \begin{cases}0, & \frac{3 T_{s}}{4} \leq t \leq T_{s}, \\
\sqrt{\frac{4}{3 T_{s}},} & \text { otherwise, }\end{cases}
\end{gathered}
$$

where $\delta=3 / 4$ is the duty cycle. The signaling structures of $\varphi_{0}(t)$ and $\varphi_{1}(t)$ are shown in Figures 5(a) and 5(b), respectively. The position of the off slot of duration $T_{s} / 4$ is changed to transmit different bits. For 2-DIPPM, the data that is estimated by using an ML decision rule is obtained using (5), where $r_{i} \in r=\left[r_{0}, r_{1}\right]$.

The values of $r_{0}$ and $r_{1}$ are determined, respectively, as follows:

$$
\begin{aligned}
& r_{0}=\int_{0}^{T_{s}} r(t) q_{0}(t) d t, \\
& r_{1}=\int_{0}^{T_{s}} r(t) q_{1}(t) d t,
\end{aligned}
$$

where $q_{i}(t)=\varphi_{i}(t) * h(t)$ denotes the template pulse for the autocorrelation in the matched filter. If data bit 0 is transmitted, that is, $i=0$, the received signal can be written as

$$
r(t)=\gamma P q_{0}(t)+n(t),
$$

where $\gamma=R \times H(0)$ contains the photodetector responsivity and path loss gain effects.

The correlator outputs are obtained as follows:

$$
\begin{aligned}
& r_{0}=\int_{0}^{T_{s}} r(t) q_{0}(t) d t=\gamma P+n_{0}, \\
& r_{1}=\int_{0}^{T_{s}} r(t) q_{1}(t) d t=\gamma P \alpha+n_{1},
\end{aligned}
$$

where $n_{0}$ and $n_{1}$ are the Gaussian random noise with zero mean and variance $N_{0} / 2$, respectively. The covariance of $n_{0}$ and $n_{1}$ is given as

$$
E\left\{n_{0}, n_{1}\right\}=\frac{N_{0}}{2} \alpha .
$$

$\alpha$ is the correlation factor and is given as follows:

$$
\alpha=\int_{0}^{T_{s}} q_{0}(t) q_{1}(t) d t
$$

Let $u=r_{0}-r_{1}$ be a random variable defined to analyze the performance of 2-DIPPM. The conditional mean and variance of $u$ are, respectively, obtained as

$$
\begin{aligned}
E\{u \mid i=0\} & =E\left\{r_{0}\right\}-E\left\{r_{1}\right\}=\gamma P(1-\alpha), \\
\operatorname{Var}\{u \mid i=0\} & =N_{0}(1-\alpha) .
\end{aligned}
$$

The ML scheme determines the correlator output element to be transmitted in the signal within $\left\{r_{z}\right\}_{z=0}^{m-1}$, which has the highest value. The error probability is calculated as

$$
P_{\mathrm{e} \mid i=0}=P(u<0 \mid i=0)=\int_{-\infty}^{0} p_{u}(u \mid i=0) d u .
$$




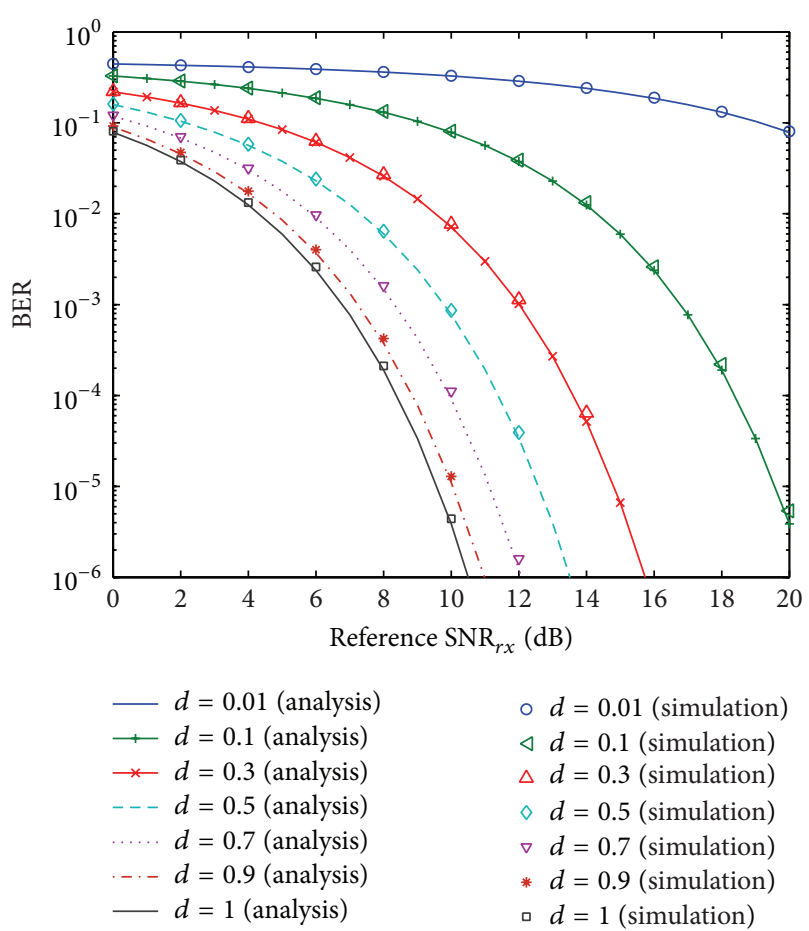

FIGURE 6: BER performance of 2-DIPPM.

The conditional probability density function of $u$ is expressed as

$$
\begin{aligned}
& p_{u}(u \mid i=0) \\
& \quad=\frac{1}{\sqrt{2 \pi N_{0}(1-\alpha)}} \exp \left[-\left(\frac{u-E(u)}{\sqrt{2 N_{0}(1-\alpha)}}\right)^{2}\right] .
\end{aligned}
$$

The error probability is derived from (20) and (21) as

$$
P_{\mathrm{e} \mid i=0}=\frac{1}{2} \operatorname{erfc}\left(\gamma P \sqrt{\frac{1-\alpha}{2 N_{0}}}\right),
$$

where

$$
\operatorname{erfc}(x)=\frac{2}{\sqrt{\pi}} \int_{x}^{\infty} \exp \left(-t^{2}\right) d t
$$

Similarly, the error probability for $i=1$ can be derived as

$$
P_{\mathrm{e} \mid i=1}=\frac{1}{2} \operatorname{erfc}\left(\gamma P \sqrt{\frac{1-\alpha}{2 N_{0}}}\right) .
$$

The total error probability for 2-DIPPM is computed as follows:

$$
\begin{aligned}
P_{e} & =P\{b=0\} P_{\mathrm{e} \mid i=0}+P\{b=1\} P_{\mathrm{e} \mid i=1} \\
& =\frac{1}{2} \operatorname{erfc}\left(\gamma P \sqrt{\frac{1-\alpha}{2 N_{0}}}\right) .
\end{aligned}
$$

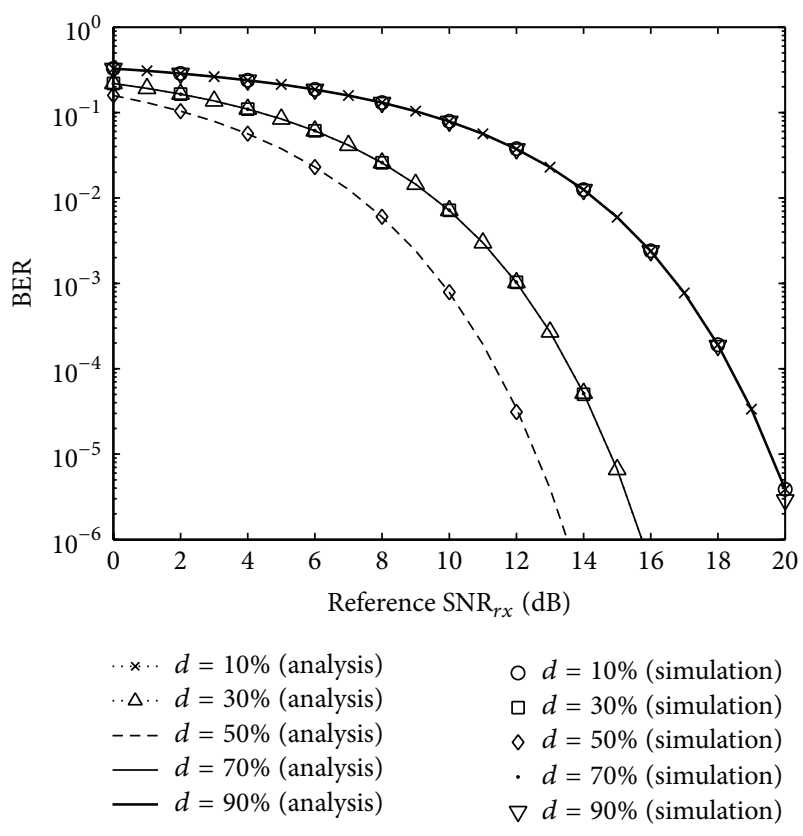

FIGURE 7: BER performance of VPPM [9].

3.2. Average Power of DIPPM. The required power means the power which is required to achieve a given BER at given bit rate. For simplicity, the Signal-to-Noise Ratio (SNR) is assumed to be high such that the BER is caused by Intersymbol Interference (ISI) by the adjacent signals. In this case, the BER is given by [4]

$$
\mathrm{BER}=\mathrm{Q}\left(\frac{d_{\min }}{2 \sigma}\right)
$$

where $d_{\text {min }}$ is the minimum Euclidean distance between any two adjacent signals which is given by $d_{\min }^{2}=\min _{i \neq j} \int\left(x_{i}(t)-\right.$ $\left.x_{j}(t)\right)^{2} d t\left(d_{\min }\right.$ should not be mixed up with dimming factor $d)$ and $\sigma^{2}$ denotes the variance of the noise $\left(\sigma^{2}=N_{0} / 2\right)$. Using $x_{0}(t)=P^{\prime} \varphi_{0}(t)$ and $x_{1}(t)=P^{\prime} \varphi_{1}(t)$ from (11), where $P^{\prime}$ is the instantaneous power under full brightness $(d=1)$, and after applying some simple calculations, the minimum Euclidean distance for 2-DIPPM can be given as

$$
d_{\min }=\frac{P^{\prime}}{\sqrt{2 \delta}},
$$

where $\delta$ is the duty cycle given in (10). Let $X^{\prime}(t)$ denote the instantaneous optical power under full brightness; then it must satisfy [4]

$$
P_{\text {avg }}=d\left\langle X^{\prime}(t)\right\rangle,
$$

where $P_{\text {avg }}$ is the required average power to achieve the desired dimming $d$ and $\langle x(t)\rangle=\lim _{T \rightarrow \infty}(1 / T) \int_{0}^{T} x(t) d t$.

Substituting $X^{\prime}(t)=P^{\prime} \varphi_{i}(t)$ into (28) and rearranging we get the following result:

$$
P^{\prime}=\frac{4 P_{\text {avg }}}{3 d} \sqrt{\frac{\delta}{R_{b}}} .
$$




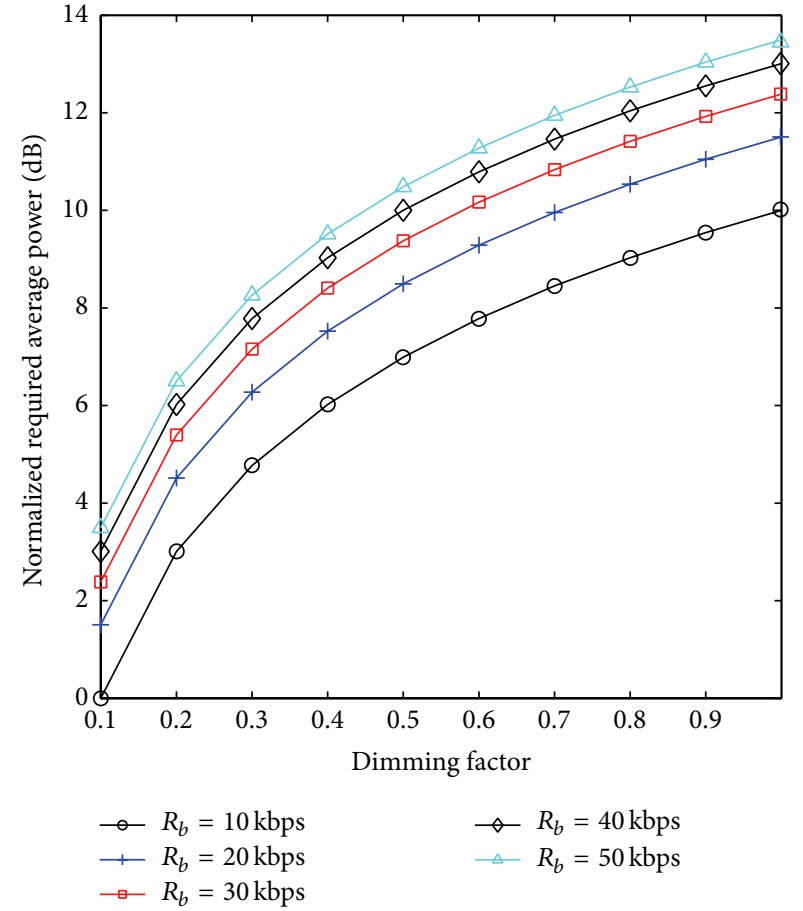

(a)

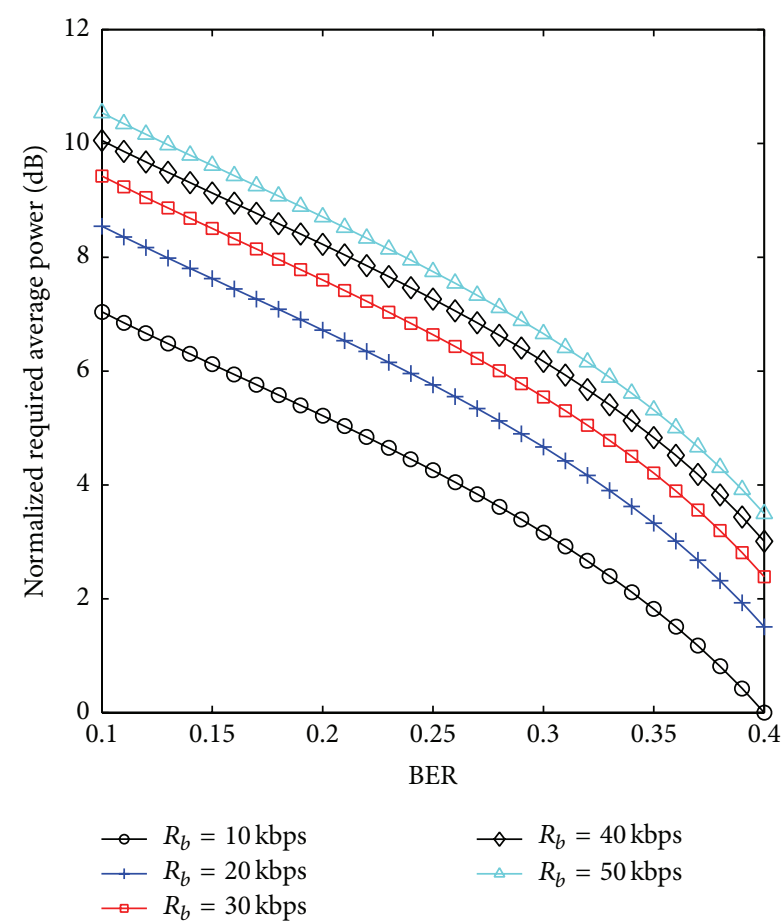

(b)

FIGURE 8: Normalized required average power according to (a) dimming factor and (b) BER when dimming factor is fixed as 0.8 .

Substituting $P^{\prime}$ from (29) into (27) gives

$$
d_{\min }=\frac{4 P_{\mathrm{avg}}}{3 d} \sqrt{\frac{1}{2 R_{b}}} .
$$

From (26) and (30) we can get

$$
P_{\mathrm{avg}}=\frac{3 d \sqrt{R_{b} N_{0}}}{2} Q^{-1}(\mathrm{BER}) .
$$

\section{Simulation Results}

To show the effectiveness of the proposed scheme, in this section, we present analytical and simulation results for 2DIPPM where a nondirected LOS channel is considered, that is, number of channel clusters $C=1$ with a symbol duration $T_{s}=0.01 \mu \mathrm{s}$.

Figure 6 shows the error performance of the 2-DIPPM. The error performance is described as the bit error rate with respect to the reference $S N R$. The reference $S_{N R}$ that is received varies by changing the dimming factor with the LOS channel. The figure shows a resemblance between the analytical and simulation results, and hence the analytical results are considered to provide a good estimation of the performance when the system parameters have been selected. The reference $\mathrm{SNR}_{r x}$ on the $x$-axis indicates the magnitude of the SNR that is obtained from the noise power attained by keeping the signal power with a 0.5 dimming factor. The figure also shows the BER results for different dimming levels, ranging from 0.01 to 1 . The dimming level is controlled via symbol energy. An increase in the dimming level is observed to improve the performance of the system since the correlation factor is constant for all dimming levels, keeping the bit resolution unchanged. The signal with a 1 dimming factor offers the best performance while a dimming factor of 0.01 exhibits the worst performance.

The IEEE considers VPPM as the best modulation scheme for VLC because of its potential to control dimming effectively as compared to other modulation schemes. Therefore, in this paper, we have compared the performance of the proposed scheme with that of VPPM [9]. Figure 7 shows both analytical and simulated BER performance of VPPM under LOS channel. From the figure it is observed that the performance of VPPM can be improved with the increase in the dimming when the dimming is below $50 \%$. On the other hand, the increasing of dimming above $50 \%$ dimming factor degrades the BER performance of the VPPM. From Figure 6, however, it is noteworthy that increasing the dimming factor even above $50 \%$ can improve the BER performance in the case of DIPPM. The dimming factor 1 in the case of DIPPM gives the best BER performance but it is not in the case of VPPM.

Figure 8 shows the analytical results of normalized required average power based on (31). Figure 8 (a) gives the analytical results of normalized required average power according to dimming factor. It can be seen that increase in the dimming factor results in increasing the required average power. Figure 8 (b) shows that the normalized average power decreases as the BER increases when the dimming level is fixed as 0.8 . The increase in the bit rate causes the average power to be increased. 


\section{Conclusion}

In this paper, Double Inverse Pulse Position Modulation (DIPPM), a new modulation scheme, is proposed for a VLC system with a LOS channel, and its performance is analyzed. Mathematical models for the transmitter, optical wireless channel, and receiver structure of DIPPM are described, and the performance of 2-DIPPM is analyzed from an analytical perspective and through simulation. The relationship between the dimming level and error performance is presented according to the channel condition. An increase in the dimming level improves the performance of the DIPPM, and as a result the proposed scheme is shown to support high dimming levels to simultaneously provide illumination and data communications. Furthermore, the performance of 2DIPPM is compared with the performance of VPPM. The comparative analysis shows the effectiveness of our proposed scheme at dimming factor above $50 \%$ where the performance of VPPM degrades. The required average power is also analyzed for DIPPM with the variations in the given BER and dimming factor for different bit rates.

\section{Conflict of Interests}

The authors declare that there is no conflict of interests regarding the publication of this paper.

\section{Acknowledgment}

This research was supported by Basic Science Research Program through the National Research Foundation of Korea (NRF) funded by the Ministry of Education (2013R1A1A2063779).

\section{References}

[1] Z. Ghassemlooy, W. Popoola, and S. Rajbhandari, Optical Wireless Communications-System and Channel Modelling with MATLAB, CRC Press, Taylor \& Francis, 2013.

[2] S. Rajagopal, R. D. Roberts, and S.-K. Lim, "IEEE 802.15.7 visible light communication: modulation schemes and dimming support," IEEE Communications Magazine, vol. 50, no. 3, pp. 72-82, 2012.

[3] M. Oh, "A flicker mitigation modulation scheme for visible light communications," in Proceedings of the 15th International Conference on Advanced Communication Technology: Smart Services with Internet of Things (ICACT '13), pp. 933-936, January 2013.

[4] K. Lee and H. Park, "Modulations for visible light communications with dimming control," IEEE Photonics Technology Letters, vol. 23, no. 16, pp. 1136-1138, 2011.

[5] K. Choi, Y. Jang, J. Noh, M. Ju, and Y. Park, "Visible light communications with color and dimming control by employing VPPM coding," in Proceedings of the 4th International Conference on Ubiquitous and Future Networks (ICUFN '12), pp. 10-12, IEEE, Phuket, Thailand, July 2012.

[6] J. M. Kahn and J. R. Barry, "Wireless infrared communications," Proceedings of the IEEE, vol. 85, no. 2, pp. 265-298, 1997.

[7] J. R. Barry, J. M. Kahn, W. J. Krause, E. A. Lee, and D. G. Messerchmitt, "Simulation of multipath impulse response for indoor wireless optical channels," IEEE Journal on Selected Areas in Communications, vol. 11, no. 3, pp. 367-379, 1993.

[8] X. Zhang, K. Cui, M. Yao, H. Zhang, and Z. Xu, "Experimental characterization of indoor visible light communication channels," in Proceedings of the 8th International Symposium on Communication Systems, Networks and Digital Signal Processing (CSNDSP '12), pp. 1-5, July 2012.

[9] J.-H. Yoo and S.-Y. Jung, "Modeling and analysis of variable PPM for visible light communications," EURASIP Journal on Wireless Communications and Networking, vol. 2013, article 134, 6 pages, 2013.

[10] B. Sklar, Digital Communications-Fundamentals and Applications, Pearson Education, 2nd edition, 2002. 

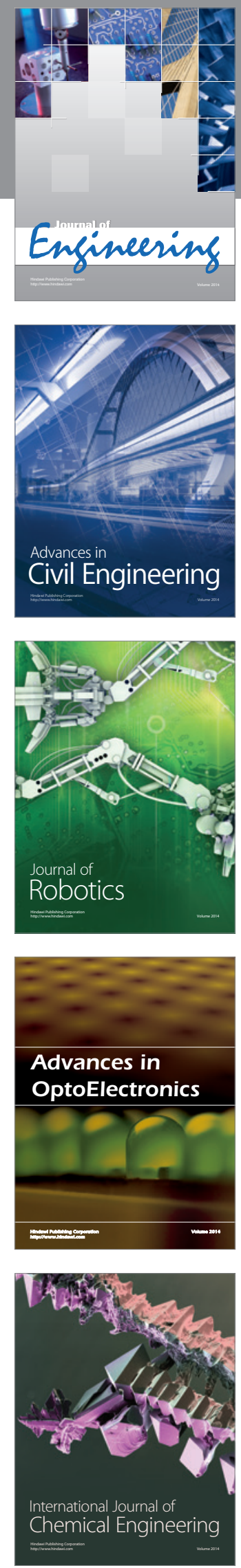

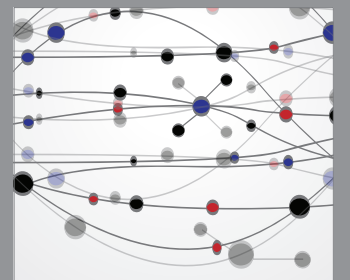

The Scientific World Journal
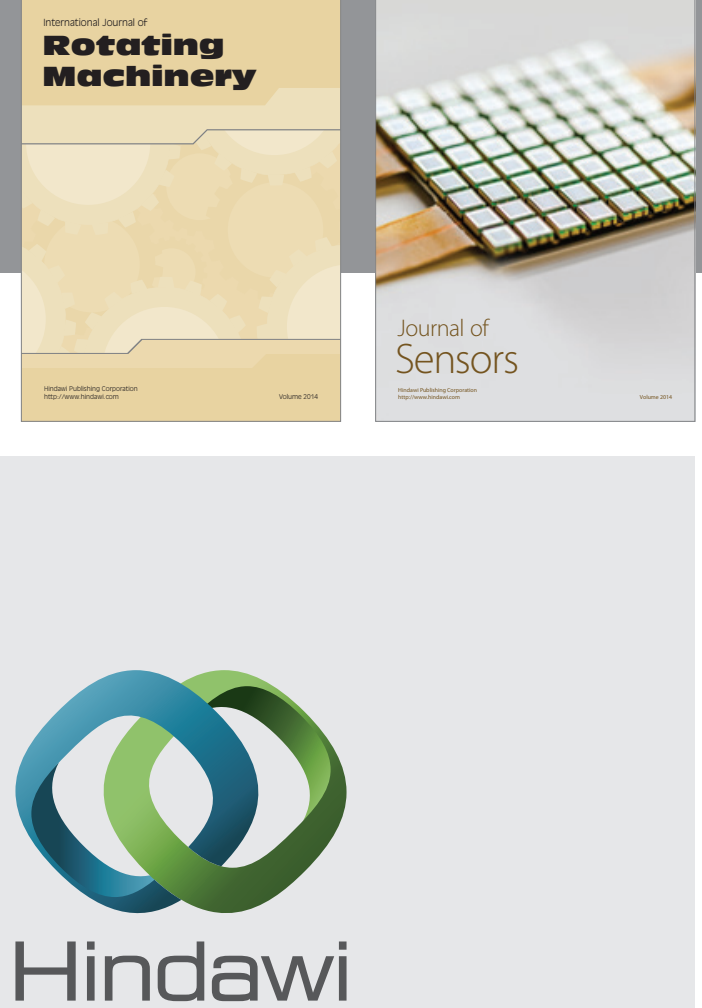

Submit your manuscripts at http://www.hindawi.com
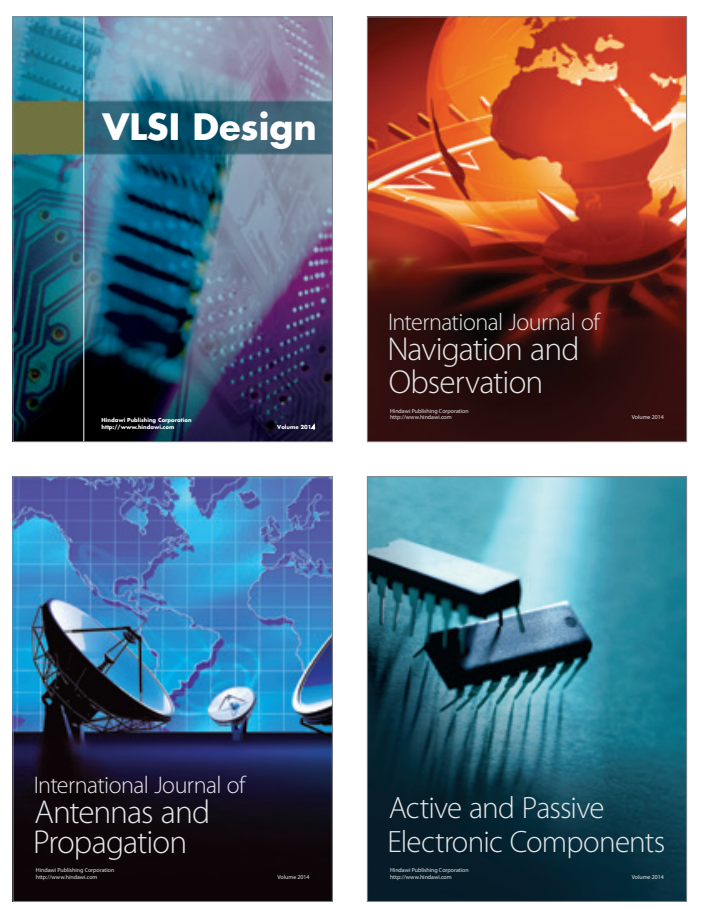
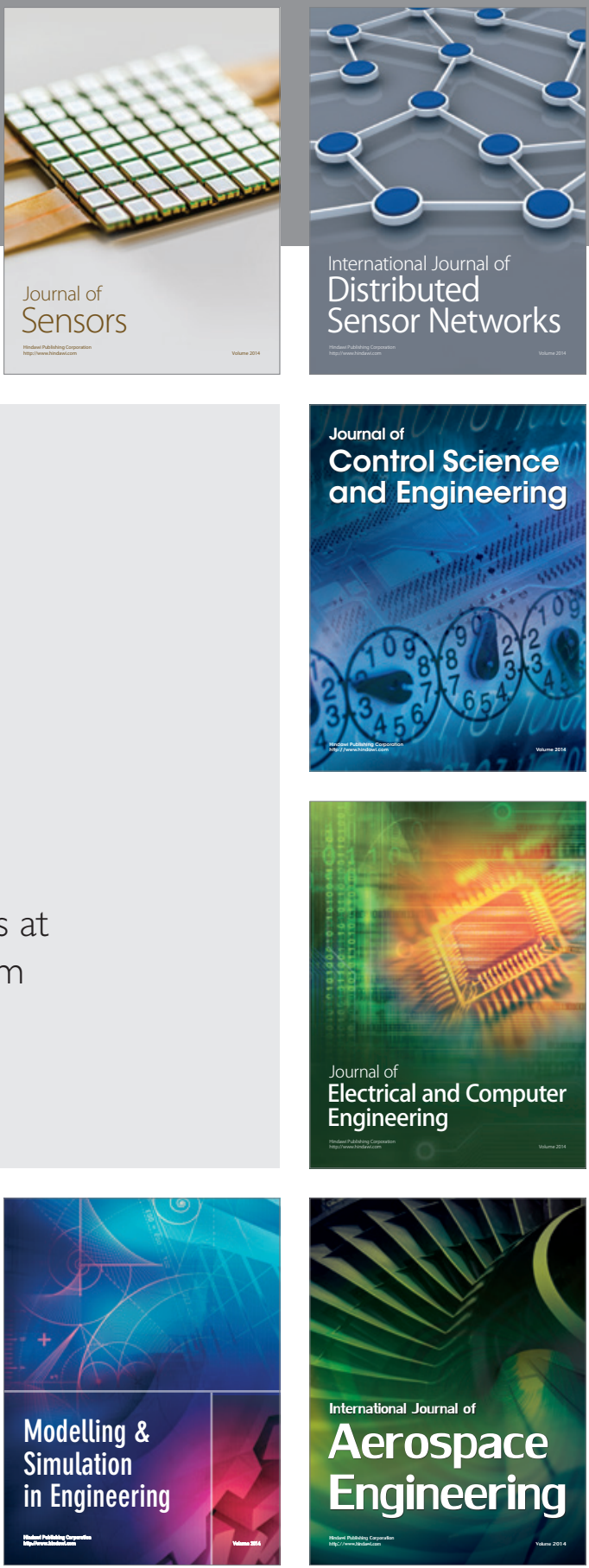

Journal of

Control Science

and Engineering
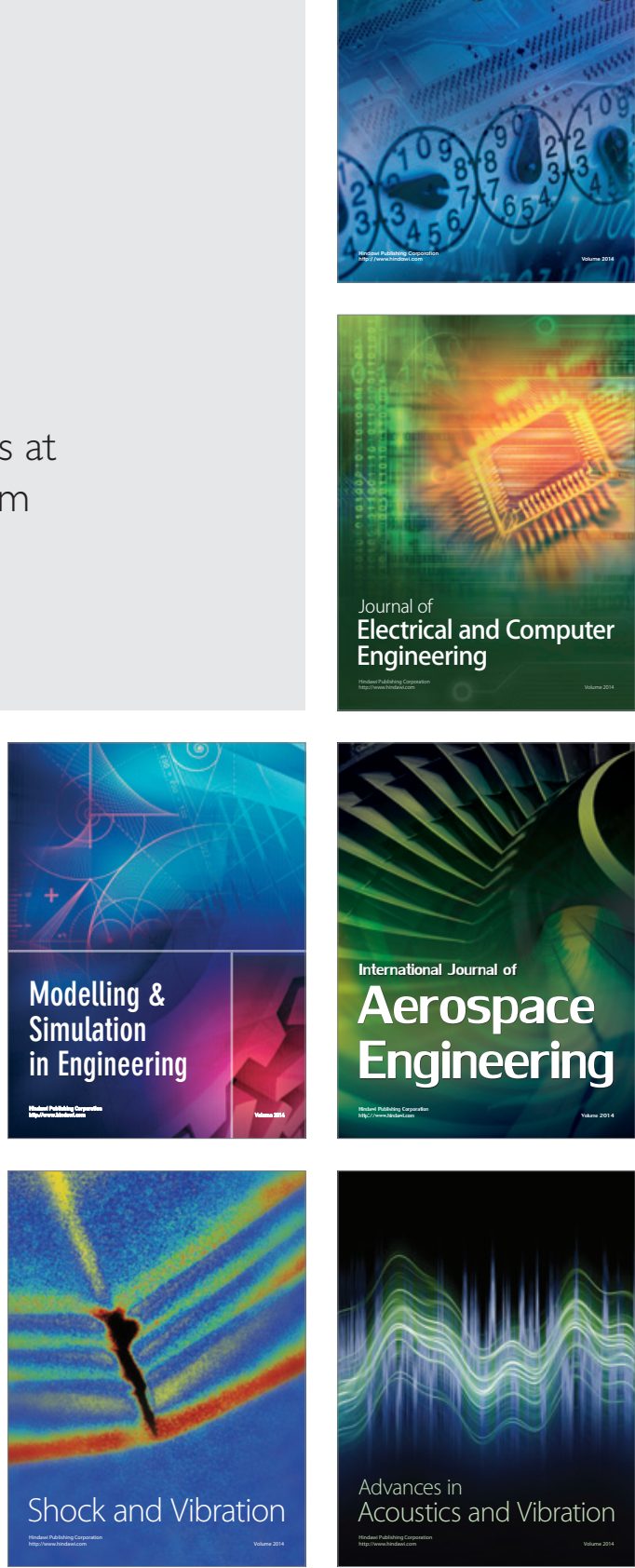